\title{
Different degrees of ischaemic injury in the right and left ventricle in cases of severe, nonfatal, pulmonary embolism
}

\author{
T. Fracasso • B. Schrag • S. Sabatasso • J.A. Lobrinus • \\ A. Schmeling $\cdot$ P. Mangin
}

Received: 29 July 2014 / Accepted: 6 October 2014 /Published online: 17 October 2014

(C) Springer-Verlag Berlin Heidelberg 2014

\begin{abstract}
Pulmonary fat embolism (PFE) is a common complication of blunt force traumas with bone fractures. Severe forms cause impedance to right ventricular (RV) ejection, with eventual right heart ischaemia and failure. In a prospective study, we have investigated 220 consecutive autopsy cases ( 73 females, 147 males, mean age 52.1 years, min 14 years, max 91 years). PFE was detected in 52 cases that were divided into three groups according to the degree of PFE (1-3). A fourth group of cases of violent death without PFE was used for comparison. In each case, histology (H\&E, Masson) and immunohistochemistry (fibronectin and C5b-9) were performed on six cardiac samples (anterior, lateral and posterior wall of both ventricles). The degree of cardiac damage was registered in each sample and the mean degree of damage was calculated in each case at the RV and left ventricle (LV). Moreover, a parameter $\Delta$ that is the difference between the
\end{abstract}

Electronic supplementary material The online version of this article (doi:10.1007/s00414-014-1096-8) contains supplementary material, which is available to authorized users.

T. Fracasso $\cdot$ S. Sabatasso $\cdot$ P. Mangin

University Center of Legal Medicine, Lausanne-Geneva, Geneva

University Hospitals, Geneva, Switzerland

B. Schrag

Service of Legal Medicine, Valais Hospital, Sion, Switzerland

J. Lobrinus

Clinical Pathology Service, Geneva University Hospitals, Geneva, Switzerland

A. Schmeling

Institute of Legal Medicine, Münster University Hospital, Münster, Germany

\section{T. Fracasso $(\bowtie)$}

Centre Universitaire Romand de Médecine Légale, Hôpitaux Universitaires de Genève, Rue Michel-Servet 1, 1211 Geneva, Switzerland

e-mail: Tony.Fracasso@hcuge.ch mean damage at the RV and the LV was calculated in each case. The results were compared within each group and between the groups. In the present study, we could not detect prevalent RV damage in cases of high degree PFE as we did in our previous investigation. In the group PFE3 the difference of the degree of damage between the RV and LV was higher than the one observed in the groups PFE0-2 with the antibody anti-fibronectin. Prevalent right ventricular stress in cases of severe PFE may explain this observation.

Keywords Right ventricle $\cdot$ Ischema $\cdot$ Fat embolism · Forensic pathology

\section{Introduction}

Pulmonary fat embolism (PFE) is a common complication of blunt force injuries occurring in major traumas, especially if fractures of long bones are present. In cases of fulminating PFE, the sudden massive obstruction of pulmonary arteries causes rapid and often lethal increase in the impendence to right ventricular (RV) ejection, with subsequent right heart ischaemia and failure [1].

We have recently proposed a method [2] to evaluate the occurrence of prevalent $\mathrm{RV}$ ischaemia determining acute right heart failure in cases of severe PFE. This method allows the diagnosis of primary right heart failure due to acute persistent pulmonary obstruction. The major limits of this previous work are bound to its retrospective character: in relatively few cases of PFE, immunohistochemical investigations were performed on available paraffin-embedded blocks of cardiac tissue, collected at autopsy on the basis of a routine sampling protocol without an extensive systematic investigation of different cardiac regions. Furthermore, the majority of the investigated cases were fatal PFE so that we could not evaluate if there is a correlation between RV damage and increasing PFE severity. 
We therefore decided to perform a prospective study whose objective is to evaluate whether RV damage increases with increasing degrees of PFE and it is homogeneously distributed in different cardiac regions.

\section{Materials and methods}

In a prospective study, we investigated 220 consecutive autopsy cases ( 73 females, 147 males, mean age 52.1 years, min 14 years, max 91 years) performed at the University Center of Legal Medicine in Geneva between July 2010 and March 2012. In each case, six cardiac regions (anterior, lateral and posterior wall of the right and the left ventricle) were sampled, fixed in $4 \%$ neutral buffered formaldehyde for $24-48 \mathrm{~h}$, and standard histology staining (haematoxylin and eosin, Masson's trichrome) were performed. Moreover, immunohistochemical reactions with the antibodies anti-fibronectin (polyclonal rabbit anti-human, Dako GmbH, Hamburg, Germany, diluted 1:2000) and anti-C5b-9 (polyclonal chicken anti-human, Quidel Corporation, San Diego, CA, diluted 1:2000) were performed in every sample. For both protocols, the streptavidin/biotin immunoperoxidase detection system (Vector Labs) was used and 3-amino-9-ethylcarbazole (AEC) was used as chromogenic substrate, according to manufacturer's instructions, with haematoxylin counterstaining. Antigen retrieval was performed for both antibodies, by incubating the slides with proteinase $\mathrm{K}$ for $5 \mathrm{~min}$ at room temperature. In order to confirm the specificity of the immunostaining, phosphate-buffered saline was substituted for the primary antibody in both procedures.

Haematoxylin and eosin-stained lung samples (five in each case, one from each lobe) were investigated for the presence of bone marrow embolism.

PFE was determined in frozen sections of the lungs (one sample from each lobe was collected and investigated). The frozen sections were stained by Oil red $\mathrm{O}$.

Classical histological signs of cardiac ischaemic damage, such as hypereosinophilia, contraction bands, fragmentation of cardiomyocytes and myocytolysis, were systematically searched and classified into four degrees $(0-$ absent, $1-$ weak, 2-moderate and 3-severe).

Coronary artery disease and myocardial fibrosis were taken into account as parameters of chronic cardiac pathology and classified in the same way.

Immunohistochemical reactions were classified into four degrees $(0-$ negative reaction, $1-$ single cell reaction, 2group cell reaction and 3 - diffuse reaction).

The degree of PFE was determined following the method proposed by Falzi [3] classified into three groups (PFE1, PFE2 and PFE3). Moreover, a fourth group constituted by cases of violent death without PFE (PFE0) was selected as a negative control.
The degree of cardiac damage was registered in each sample and the mean degree of damage was calculated in each case at the RV and left ventricle (LV). Moreover, a parameter $\Delta$ that is the difference between the mean damage at the RV and the LV was calculated in each case.

The mean degree of damage at both ventricles was compared within each group. Furthermore, the mean degree of damage at each ventricle as well as the parameter $\Delta$ was compared between the groups.

Student's $t$ test and analysis of variance were performed for statistical analysis. $p$ values $\leq 0.05$ were considered as statistically significant.

\section{Results}

Demographic data

PFE was observed in 52 out of 220 cases $(23.6 \%, 16$ females, 36 males, mean age 56 years, min 18 years, max 89 years). In six cases in which PFE occurred, myocardial ischaemia/ infarction was diagnosed; these cases were therefore excluded from this study. The remaining 46 cases were distributed into three groups according to the degree of PFE. Demographic data and distribution of the causes of death are given in Table 1. The groups are described in more detail in tables 14 ESM.

\section{Survival time}

Information on circumstances of death and body recovery was available in every case. Amongst the individuals included in the study, 19 persons $(27.5 \%)$ were still alive when discovered (four in PFE1, ten in PFE2, five in PFE3 and zero in PFE0). In this subgroup, the median survival time (time between recovery and death) was $11.6 \mathrm{~h}$ (min. $20 \mathrm{~min}$, max. $96 \mathrm{~h}$, median $3 \mathrm{~h}$ ). Only four patients survived longer than $6 \mathrm{~h}$. Because of very limited data, groups were not compared for this parameter.

\section{Traumatic lesions}

Detail on traumatic lesions is given in Tables 1ESM-4ESM. The distribution of lesion is similar in the groups PFE1, PFE2 and PFE3. Cases included in the group PFE0 globally showed less extended lesions. Dissection of soft tissues of the back of the body and extremities was systematically performed in cases of polytrauma (i.e. fall from height, traffic accident, fall down stairs), homicide, death in custody and death after fight. This technique was not systematically performed in cases of suicide. We cannot then exclude that in group PFE0, where suicide represents the most frequent manner of death, 
Table 1 Demographic data and causes of death in the investigated cases

\begin{tabular}{lllll}
\hline & PFE0 & PFE1 & PFE2 & PFE3 \\
\hline$N$ & 21 (5 females, 16 males) & 16 (6 females, 10 males) & 18 (3 females, 15 males) & $12(3$ females, 9 males $)$ \\
Mean age (years) & 57 & 54 & 52 & 63 \\
Blunt force injury & 6 & 5 & 7 & 9 \\
Gunshot & 5 & 3 & 1 & 0 \\
Sharp force injury & 2 & 0 & 2 & 0 \\
Asphyxia & 8 & 3 & 3 & 2 \\
Other & 0 & 5 & 5 & 1 \\
\hline
\end{tabular}

traumatic lesions were underdetected. Groups were therefore not compared for this parameter.

\section{Histology}

Minimal bone marrow embolism (less than one embolus per sample) was observed in three cases in PFE1, three cases in PFE2 and four cases in PFE3. Bone marrow emboli were not observed in PFE0. These differences are not statistically significant.

Fragmentation of cardiomyocytes was more observed at the LV compared to the RV in the group PFE0 $(p<0.05)$.

Contraction bands were more observed at the RV than the LV in the group PFE2 $(p<0.05)$.

In the group PFE1, coronary artery disease and myocardial fibrosis were mostly detected at the $\operatorname{LV}(p<0.05)$.

Coronary artery disease was more detected at the LV in the group PFE3 $(p<0.05)$.

Fragmentation was more observed at the LV in PFE0 than PFE1 and PFE3 $(p<0.05)$.

Contraction bands were more detected at the RV in PFE1 than PFE2 $(p<0.05)$.

Other comparisons within and amongst the groups showed no statistically significant results.

\section{Immunohistochemistry}

The comparisons of the antibody expression within each group showed no significant differences between the RV and LV.

Concerning the comparison between the groups, the following significant results were observed:

Fibronectin was more expressed in the LV in PFE0 than PFE3 $(p<0.05)$.

The complement complex C5b-9 showed a similar expression in all the groups.

The comparison of the parameter $\Delta$ for the antibody antifibronectin showed that the RV was significantly more damaged in comparison to the LV in group PFE3 compared to PFE0, PFE1 and PFE2.
The analysis of variance showed that the distribution of myocardial damage detected by immunohistochemistry was similar in the investigated cardiac regions in each group.

\section{Discussion}

Sex and age distribution was similar in the four groups. Moreover, the degree of coronary artery disease and myocardial sclerosis, indicating the baseline cardiac pathology of the patients included in this study, was also similar. The groups could then be compared to each other.

The systematic search for classical histological signs of myocardial ischaemia gave few incoherent statistically significant results whose interpretation is tricky. These signs are regularly observed in cases of myocardial infarction [4]; their specificity, especially if their distribution is patchy, like in this study, is however low [5]. Similarly to pulmonary thromboembolism, patients surviving PFE only rarely develop frank $\mathrm{RV}$ infarction [6]; moreover, approximately $6 \mathrm{~h}$ of ischaemia must occur before myocardial infarction can be detected by histology [7]. In the present study, only four patients survived more than $6 \mathrm{~h}$ (data not shown), too few to draw any conclusion.

Differently from our previous study [2], in the present investigation, we could not observe statistically significant prevalent RV damage in cases of high degree PFE by means of immunohistochemistry. If one considers all the cases included in the groups PFE1-3 in the actual study, fatal PFE occurred in $2.5 \%$ (one case), while in the previous retrospective study we performed, its prevalence was much higher (38\%). This difference (due to different selection criteria) probably explains the different results: in cases of violent death due to polytrauma, even if an important PFE occurs, the RV firstly reacts with compensative mechanisms (dilatation and increased coronary perfusion) against the stress induced by sudden increase of the pulmonary impedance [8]. This compensative mechanisms delay the beginning of myocardial ischaemia so that these patients probably die before developing frank RV ischaemia. Moreover, the 
Table 2 The mean degree and frequency of expression of the antibodies anti-fibronectin and C5b-9 in the different groups and $\Delta$ values

\begin{tabular}{|c|c|c|c|c|c|c|c|c|c|c|c|c|}
\hline & \multicolumn{3}{|l|}{ PFE0 } & \multicolumn{3}{|l|}{ PFE1 } & \multicolumn{3}{|l|}{ PFE2 } & \multicolumn{3}{|l|}{ PFE3 } \\
\hline & $\mathrm{RV}$ & $\mathrm{LV}$ & $\Delta$ & $\mathrm{RV}$ & $\mathrm{LV}$ & $\Delta$ & $\mathrm{RV}$ & LV & $\Delta$ & $\mathrm{RV}$ & LV & $\Delta$ \\
\hline Fibronectin \% & $1.57(86)$ & $1.60(81)$ & -0.03 & $1.27(75)$ & $1.40(88)$ & -0.13 & $1.22(89)$ & $1.20(89)$ & 0.02 & $1.39(75)$ & $0.68(75)$ & 0.61 \\
\hline C $5 b-9 \%$ & $0.51(48)$ & $0.33(33)$ & 0.18 & $0.37(56)$ & $0.35(44)$ & 0.02 & $0.48(61)$ & $0.39(50)$ & 0.09 & $0.39(42)$ & 0.08 (17) & 0.31 \\
\hline
\end{tabular}

physiopathology of polytrauma is very complex: in cases of early mortality it includes, besides PFE, organ/soft tissue injury and blood loss. In cases with late mortality, even more complex mechanisms are involved (i.e. hypotension, ischaemia-reperfusion injuries, compartment syndrome, inflammation, coagulation disorders) [9]. In such a situation, biventricular cardiac damage is highly likely to occur as it is shown by similar immunohistochemical expression of the investigated markers in the RV and LV in PFE0-2 (Table 2). The most intriguing result emerging from this study is that in PFE3, the difference of the degree of damage between the RV and LV was higher than the one observed in the groups PFE0 2 with the antibody anti-fibronectin and a similar tendency was observed with the antibody anti-C5b-9 (Table 2). Indeed, in comparison to the other groups, it is the LV that shows significant lower ischaemic damage in the group PFE3. This observation is even more intriguing if one considers that in PFE3, coronary artery disease was significantly higher at the LV than the RV, which increases ischaemic risk at this level. It might be that PFE3 changes cardiopulmonary haemodynamic in these victims who may die in a situation of right heart stress and failure more than global heart failure.

The only patient died from PFE was a 69-year-old man who showed sudden hypotension with transitory cardiac arrest shortly after surgical shoulder replacement. Echocardiography showed massive dilatation of the RV with leftward shift of the interventricular septum and dilatation and moderate regurgitation of the tricuspid valve. Pulmonary artery systolic pressure was approximately $75 \mathrm{mmHg}$. The patient was transferred to the intensive care unit where he died 2 days later. Figure 1 shows immunohistochemical reactions in the RV and $\mathrm{LV}$ in this patient.

Differently from our previous studies [2, 10-12], in the present one, we used a different antibody for the detection of C5b-9. We think that the higher sensibility of this antibody can explain the higher expression observed in cases of PFE in this study (Table 2) compared to the previous investigation (study group $19 \% \mathrm{RV}, 10 \% \mathrm{LV}$ ). We had already expressed some concerns about the low expression of the antibody we used in previous investigations and advised that our results were
Fig. 1 Expression of the antibodies anti-fibronectin and C5b-9 in the RV $(\mathbf{a}, \mathbf{b})$ and LV $(\mathbf{c}, \mathbf{d})$ in the only case of fatal PFE (degree III) observed in this study
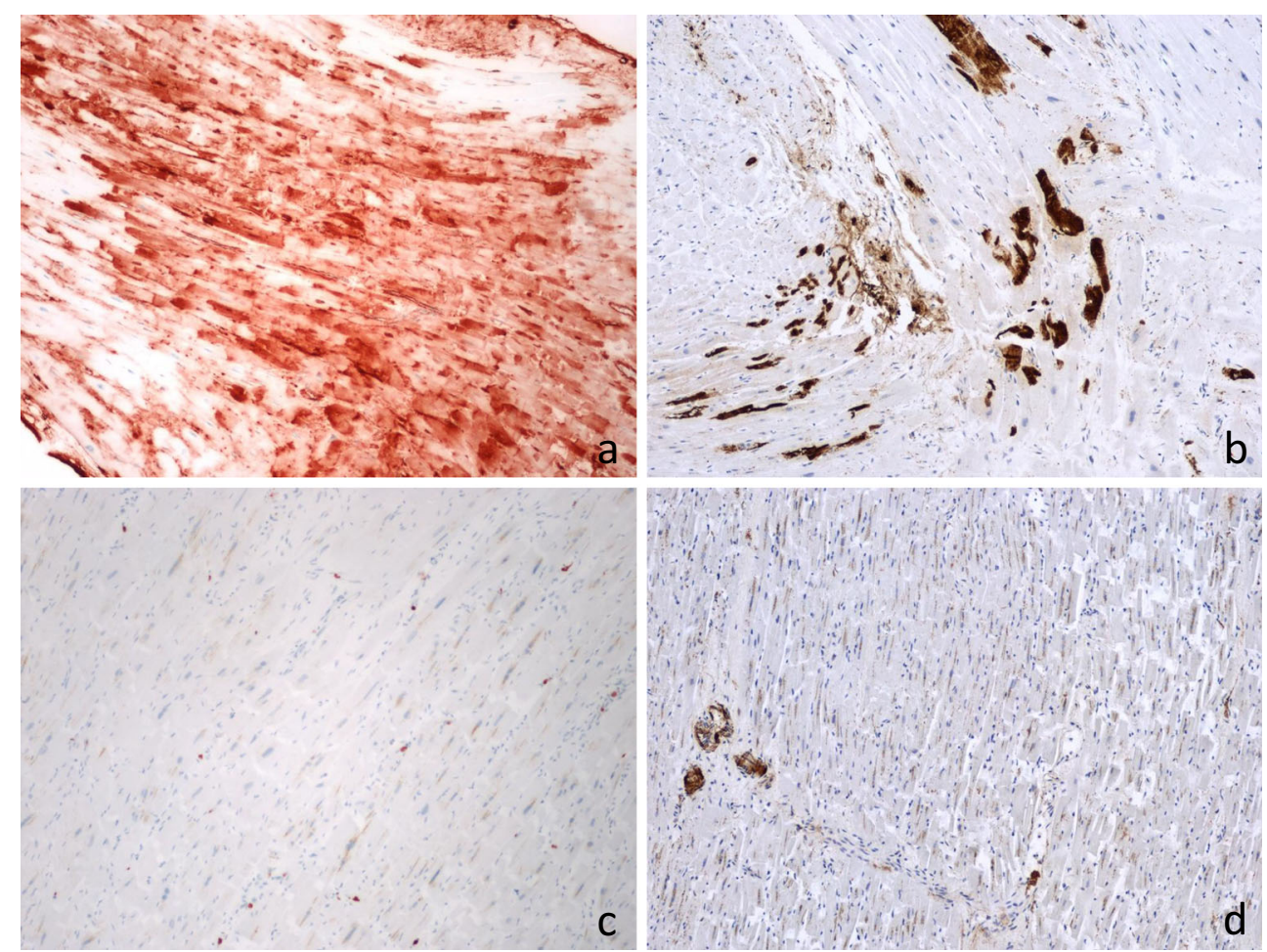
possibly influenced by difficult interpretation of low expression areas [12]. The observations of the present study support our previous interpretation.

To sum up, in the present study, we could not detect prevalent RV damage in cases of high-degree PFE as we did in our previous investigation. According to our opinion, this is due to important differences in the investigated population in which severe PFE was not the main cause of death (but in one case). Furthermore, in the majority of the cases, the time of agony was probably too short to allow substantial ischaemia to occur and subsequently to be detected by both histology and immunohistochemistry. Interestingly, in the group PFE3, the distribution of the damage was significantly different from the other groups, as ischaemic changes were mostly localized at the RV while the LV was less involved. Different haemodynamic conditions (prevalent right ventricular stress) in cases of severe PFE may explain this observation.

Acknowledgments This study was financially supported by a MIMOSA grant from the Department of Health and Community Medicine, Faculty of Medicine, University of Geneva.

\section{References}

1. Akhtar S (2009) Fat embolism. Anesthesiol Clin 27:533-550

2. Fracasso T, Karger B, Pfeiffer H, Sauerland C, Schmeling A (2010) Immunohistochemical identification of prevalent right ventricular ischemia causing right heart failure in cases of pulmonary fat embolism. Int J Legal Med 124:537-542
3. Falzi G, Henn R, Spann W (1964) Über pulmonale Fettembolien nach Traumen mit verschieden langer Überlebenszeit. Münch med Wschr 106:978-981

4. Baroldi G (2006) Meaning of different forms of structural myocardial injury. In: Fineschi V, Baroldi G, Silver MD (eds) Pathology of the heart and sudden death in forensic medicine. CRC, Boca Raton, pp 43-50

5. Dettmeyer RB (2011) Sudden coronary death. In: Dettmeyer RB (ed) Forensic histopathology. Springer, Berlin, pp 241-245

6. Dang NC, Johnson C, Eslami-Farsani M, Haywood J (2005) Myocardial injury or infarction associated with fat embolism in sickle cell disease: a report of three cases with survival. Am J Hematol 80: 133-136

7. Thygesen K, Alpert JS, Jaffe AS, Simoons ML, Chaitman BR, White $\mathrm{HD}$, The writing group on behalf of the Joint ESC/ACCF/AHA/ WHF Task Force for the Redifinition of Myocardial Infarction (2012) Universal definition of myocardial infarction. J Am Coll Cardiol 60:1581-1598

8. Fracasso T (2013) Histological and immunohistochemical investigations of acute right ventricular damage in forensic pathology. Dissertation, University of Geneva

9. Keel M, Trentz O (2005) Physiopathology of trauma. Injury 36:691709

10. Fracasso T, Pfeiffer H, Sauerland C, Schmeling A (2011) Morphological identification of right ventricular failure in cases of fatal pulmonary thromboembolism. Int J Legal Med $125: 45-50$

11. Fracasso T, Pfeiffer H, Michaud K, Köhler H, Sauerland C, Schmeling A (2011) Immunohistochemical expression of fibronectin and C5b-9 in the myocardium in cases of carbon monoxide poisoning. Int J Legal Med 125:377-384

12. Fracasso $T$, Pfeiffer $H$, Köhler $H$, Wieseler $S$, Hansen SD, Jentgens L, Sauerland C, Schmeling A (2011) Immunohistochemical expression of fibronectin and C5b-9 in the myocardium in cases of fatal ethanol intoxication. Int $\mathrm{J}$ Legal Med 125:537-542 